\title{
A highly efficient two level diamond based single photon source
}

\author{
D. A. Simpson 7 E. Ampem-Lassen, B. C. Gibson, S. Trpkovski, F. M. \\ Hossain, S. T. Huntington, A. D. Greentree, L. C. L. Hollenberg, and S. Prawer \\ Quantum Communications Victoria, School of Physics, University of Melbourne
}

\begin{abstract}
An unexplored diamond defect centre which is found to emit stable single photons at a measured rate of $1.6 \mathrm{MHz}$ at room temperature is reported. The novel centre, identified in chemical vapour deposition grown diamond crystals, exhibits a sharp zero phonon line at $734 \mathrm{~nm}$ with a full width at half maximum of $\sim 4 \mathrm{~nm}$. The photon statistics confirm the center is a single emitter and provides direct evidence of the first true two-level single quantum system in diamond.
\end{abstract}

Nanotechnology and photonics industries around the world are currently undergoing a revolution as research into individual quantum systems opens up new and exciting opportunities in quantum information processing (QIP) [1, 2, 3] and quantum key distribution (QKD) [4]. At the forefront of this revolution is the development of light sources which emit single particles of light on-demand, known as single photon sources (SPSs). Single photon generation has been demonstrated with several different technologies including quantum dots (QDs) [5], single molecules [6] and more recently carbon nano-tubes [7]; but perhaps the must promising and applicable source of single photons for practical applications arise from optical defects in diamond which can be easily accessed and operate at room temperature. To date, of the over 500 known defect centres in diamond only three have been identified as single photon (SP) emitters 8, 9, 10, 11, 12]. However, what sets these color centres apart from the other technologies is their uncompromised photo-stability at room temperature. Until now, the SP emission rates achieved in diamond based SPSs have been limited to $\sim 300 \mathrm{kHz}$ due to the presence of meta-stable/shelving states which act to reduce the efficiency of the single quantum system $[8,9,10,11]$. In this paper we report the first diamond based defect centre which behaves as a true two-level system. This centre also exhibits the highest measured $\mathrm{SP}$ emission rate at room temperature in the $\mathrm{MHz}$ regime.

Many schemes have been proposed to improve the emission rate from diamond based SPSs with perhaps the most promising involving the incorporation of single emitting diamond nano-crystals into cavity structures [13]. Unfortunately, the lack of mature cavity technology has limited the incorporation of diamond based SPSs into such designs [14]. As a result, researchers are in parallel exploring new defect centres which may result in more efficient SP emission. For diamond based SPSs to emit more efficiently new defect centres need to be explored and/or created which comprise a simple two level energy structure similar to that observed in semiconductor QDs. To this end, this work explores novel defect centres which can be created synthetically using the well known microwave plasma enhanced chemical vapour deposition (MPECVD) technique [15].

The fused silica substrate characterised in this work was $1 \times 1 \mu \mathrm{m}$ in size and ultrasonically seeded with commercial grade diamond powder $0-2 \mu \mathrm{m}$ in size prior to MPECVD growth. The 30 minute diamond growth was conducted under the conditions described in [15], with background levels of nitrogen, nickel and silicon present in the system. Photo-luminescent studies were conducted on the fused silica substrate with the aim of identifying novel luminescent defect centres. Many emitting centres were identified by the in house fibre based scanning confocal microscope, operating with 532 $\mathrm{nm}$ excitation and a spatial resolution of $\sim 400 \mathrm{~nm}$. Figure 1 (a) illustrates the large number of emitting centres within a $20 \times 20 \mu \mathrm{m}$ area of the substrate. The photo-luminescence (PL) spectra from each emitting crystal was measured in the first instance to determine the type of emitting centre and secondly to identify any uncharacteristic spectral lines. The room temperature PL spectrum from the emitting centre identified in the centre of Figure 1 (b) is shown in Figure 1 (c). The spectra reveals a sharp zero phonon line (ZPL) centered at $734 \mathrm{~nm}$ with a full width at half maximum (FWHM) of $4.1 \mathrm{~nm}$. The Huang-Rhys factor which is a measure of the relative intensity of the ZPL compared to the entire spectrum was found to be 0.81 which is higher than that reported for the nickel related (NE8) centre [8] and significantly higher than the well known nitrogen vacancy $(\mathrm{N}-\mathrm{V})$ centre $(0.04)[8]$. The photon statistics of the centre were investigated using a fibre based version of the Hanbury Brown and Twiss interferometer [16]. The second-order intensity autocorrelation function:

$g^{(2)}(\tau)=<I(t) I(t+\tau)>/<I(t)>^{2}$

obtained from the photon coincidence rate histogram is shown in Figure 2 for three different incident pump powers. The autocorrelation function minimum at the zero delay time, $g^{(2)}(0)$, was measured to be 0.16 , with the deviation from zero being attributed to the background surrounding and/or within the emitting crystal. The important conclusion to be drawn from the 

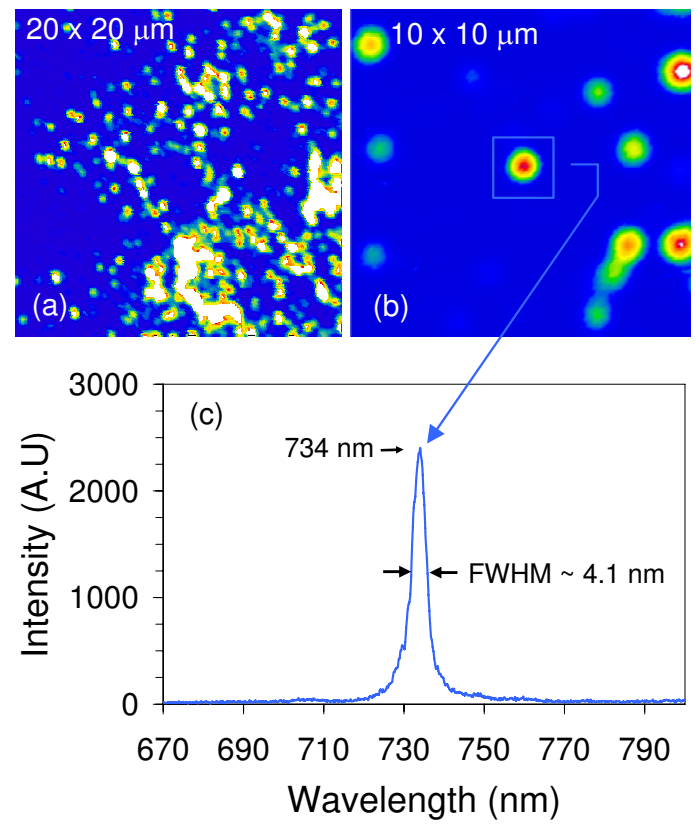

FIG. 1: (a) Fluorescence intensity confocal map of emitting diamond crystals on a fused silica substrate under $532 \mathrm{~nm}$ excitation. (b) High resolution image of a $10 \times 10 \mu \mathrm{m}$ region of the substrate. (c) The PL spectrum from the emitting crystal identified in the centre of (b).

autocorrelation measurements, as a function of pump power, is the absence of 'photon bunching' 17, 18] in the coincidence histogram. Photon bunching is observed in all of the single diamond related defects reported to date [8, 9, 10] and is attributed to the presence of a meta-stable quenching/shelving state in the electronic structure of the atomic system. The absence of photon bunching in the coincidence histogram for excitation powers well above saturation is direct evidence of a two level energy scheme. The power dependence of the second order correlation function based on a two level energy scheme can be described by $[9]$ :

$$
g^{(2)}(\tau) \simeq 1-\exp ^{\left(-k_{T} t\right)}
$$

where $k_{T}=k_{21}+k_{12}$, with $k_{12}$ and $k_{21}$ being the pumping rate and photon emission rate of the excited state, respectively.

A least squares fit of Eq.(11) was applied to the measured anti-bunching data as a function of pump power, as seen in Figure 2. The fitting parameter $k_{T}$ can then be used to obtain the fluorescence decay rate of the excited state as $k_{T}(0)=k_{21}$, in the limit of zero pump excitation $(P \rightarrow 0)$. Figure 3 shows the fitting parameter $k_{T}$ as a function of excitation power. From the applied linear fit to the data the excited state lifetime $\tau_{2}=1 / k_{21}$ $=13.6 \mathrm{~ns}$. This lifetime is comparable to the $(\mathrm{N}-\mathrm{V})$ centre in bulk diamond (11 ns) [19] and considerably

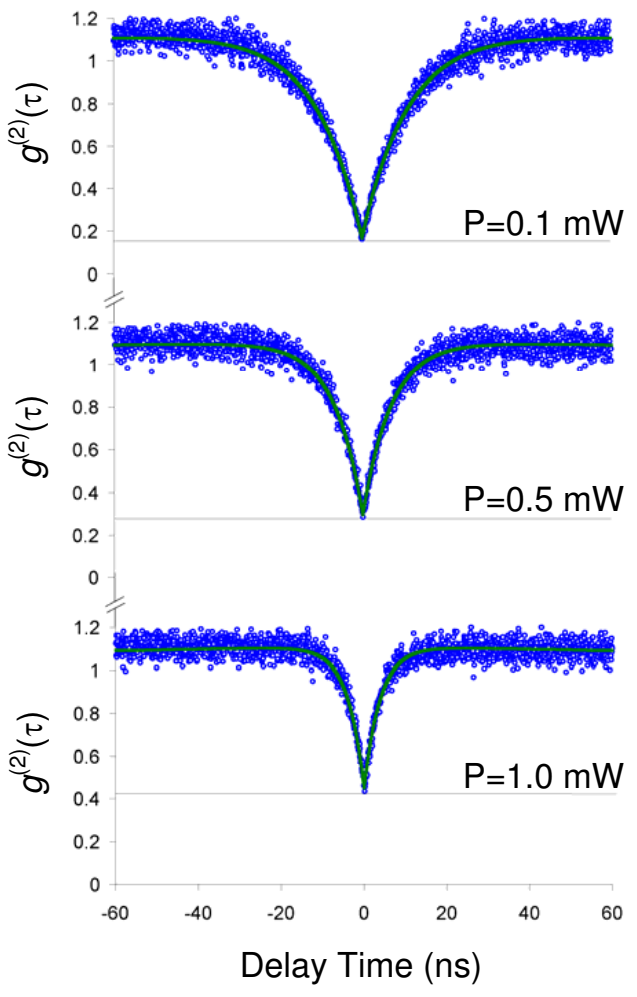

FIG. 2: Anti-bunching data from a single $734 \mathrm{~nm}$ defect centre for excitation powers ranging from $P=0.1-1.0 \mathrm{~mW}$. The dots represent the measured coincidences whilst the solid line was obtained from a theoretical fit as described in the text. The pronounced dip in the coincidence counts at delay time $t=0$ ns, illustrates the sub-Poissonian statistics of the source.

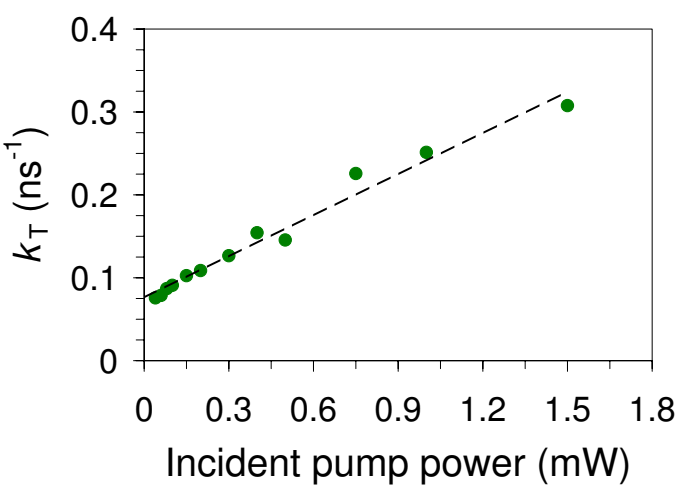

FIG. 3: Decay rate $k_{T}$ as a function of incident pump power. The solid line represents a linear fit to the data as the fluorescence decay rate is linearly dependent on the pump excitation.

longer than those observed from the (NE8) (2 ns) [17] and silicon-vacancy (Si-V) (1.2 ns) [10] centres; however due to the two level nature of the atomic centre it is able to emit more efficiently compared to its three level system counterparts. This is evidenced by the extraordinary single photon emission rate (see Figure 4) measured as a function of pump power, under $532 \mathrm{~nm}$ 
excitation. The saturation behaviour of the emission

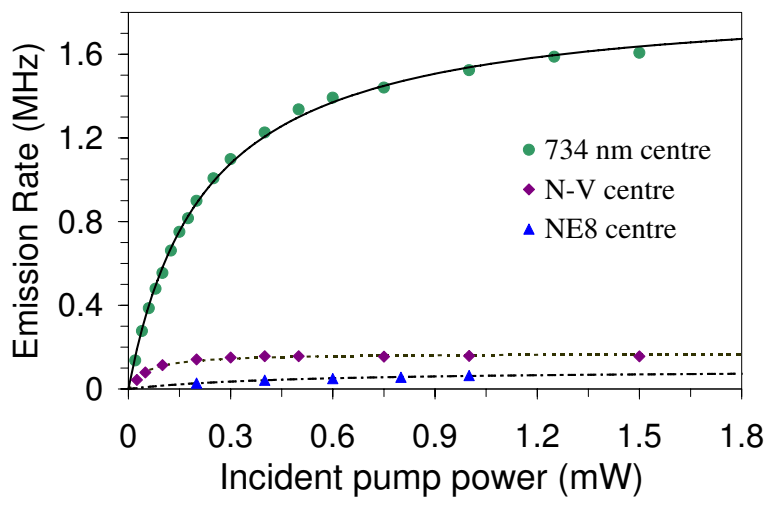

FIG. 4: Photon emission rate for the $734 \mathrm{~nm}$ single emitting centre as a function of pump power when excited at $532 \mathrm{~nm}$. The emission rate is given by the sum of the counts on the two APDs in the Hanbury Brown and Twiss arrangement. The fit to the measured data is discussed in the text. The measured emission rates for a single N-V and NE8 centre are included in the figure as a comparison and were all measured using the same experimental setup, with the exception of the NE8 data which was measured under $685 \mathrm{~nm}$ excitation.

is consistent with that expected for a single atomic system with a finite lifetime. The theoretical fit to the measured emission data was derived from the steady state solution of the excited state population of a two level system and is given by: $I(P)=I_{\text {sat }} P /\left(P_{\text {sat }}+P\right)$, where $I_{\text {sat }}$ represents the photon saturation count rate, $P$ is the incident pump power and $P_{\text {sat }}$ is the saturation pump power. From the fit to the experimental data $P_{\text {sat }}=224 \mu \mathrm{W}$ and $I_{\text {sat }}=1.8$ million counts $/ \mathrm{s}$. The saturation power is comparable to that found for $\mathrm{N}-\mathrm{V}$ centres and considerably less than that observed for the NE8 and $\mathrm{Si}-\mathrm{V}$ centres. Moreover, the saturation count rate of the $734 \mathrm{~nm}$ centre is the highest reported for a single diamond defect and the first observation of stable $\mathrm{MHz}$ emission from a single photon source operating at room temperature. The two level nature of the centre also allows an accurate estimate of the total collection efficiency of the system to be made as there is no quenching state reducing the number of emitting photons. Based on the fluorescence lifetime, the centre emits at $73.5 \mathrm{MHz}$ of which $1.6 \mathrm{MHz}$ was detected; this equates to a total system collection efficiency of $2.17 \%$. With further optimization of the substrate and the potential to manipulate diamond crystals onto plasmonic structures [20], there is the possibility to achieve SP emission into the tens of $\mathrm{MHz}$ range at room temperature.

The atomic structure of the single emitting defect centre is at this stage unclear, there are reports of a 733 $\mathrm{nm}$ absorption peak from $\mathrm{Si}$ related centres in single crystal diamond, however no emission peaks from the
$733 \mathrm{~nm}$ line have been observed in the PL under $514 \mathrm{~nm}$ excitation [21]. One can speculate though that the origin of the centre lies within a mixture of the constituents present in the diamond reactor during growth which includes nitrogen, silicon, nickel and hydrogen. PL studies of the diamond seed material before growth under $532 \mathrm{~nm}$ excitation revealed $\mathrm{N}-\mathrm{V}$ centres were present in a number of seed crystals, however, this fluorescence emitter was only observed after being exposed to the MPECVD process.

A new and highly efficient diamond-based single photon source operating in the infra red at $734 \mathrm{~nm}$ has been fabricated and characterised. The diamond defect exhibits an intense and spectrally narrow emission line and is the first SPS to exhibit $\mathrm{MHz}$ operation at room temperature. The centre is found to significantly extend the performance range of diamond based SPSs due to its two level nature. With the opportunity to manipulate such sources onto structures, which can further enhance the emission properties, the future for practical room temperature quantum devices looks bright.

The authors gratefully acknowledge Igor Aharonovich and Stefania Castelletto for many helpful discussions. This project was supported by Quantum Communications Victoria, which is funded by the Victorian Government's Science, Technology and Innovation initiative.

* Electronic address: simd@unimelb.edu.au

[1] M. Nielsen et al., Quantum computation and quantum information. (Cambridge University Press, Cambridge 1964).

[2] J. Wrachtrup et al., J. Phys.: Condens. Matter 18, 807 (2006).

[3] A. Greentree et al., Mater. Tod. 11, 22 (2008).

[4] N. Gisin et al., Rev. Mod. Phys. 74, 145 (2002).

[5] C. Santori et al., Phys. Rev. Lett. 86, 1502 (2001).

[6] C. Brunel et al., Phys. Rev. Lett. 83, 2722 (1999).

[7] A. Hogele et al., Phys. Rev. Lett. 100, 217401 (2008).

[8] T. Gaebel et al., New J. Phys. 6, 98 (2004).

[9] C. Kurtsiefer et al., Phys. Rev. Lett. 85, 290 (2000).

[10] C. Wang et al., J. Phys. B: Atomic molecular and optical physics 39, 37 (2006).

[11] J. Rabeau et al., Appl. Phys. Lett. 86, 131926 (2005).

[12] I. Aharonovich et al., Appl. Phys. Lett. 93, 243112 (2008).

[13] C. H. Su. et al., Opt. Exp. 16, 6240 (2008).

[14] S. Strauf et al., Nature Photon. 1, 704 (2007).

[15] J. Rabeau et al., Appl. Phys. Lett. 86, 134104 (2005).

[16] R. Twiss et al., Nature 180, 324 (1957).

[17] E. Wu et al., Opt. Exp. 14, 1296 (2006).

[18] F. Jelezko et al., J. Phys. Stat. Sol. A 203, 3207 (2006).

[19] A. Beveratos et al., Phys. Rev. A 64, 061802 (2001).

[20] A. Collins et al., Nature 450, 402 (2007).

[21] I. Kiflawi et al., Diamond Relat. Mater. 6, 146 (1997). 